\title{
A NuSTAR view of powerful $\gamma$-ray loud blazars
}

\author{
G. Ghisellini ${ }^{1}$, M. Perri ${ }^{2,3}$, L. Costamante ${ }^{2}$, G. Tagliaferri ${ }^{1}$, T. Sbarrato ${ }^{4}$, S. Campitiello ${ }^{5}$, G. Madejski ${ }^{6}$, \\ F. Tavecchio ${ }^{1}$, and G. Ghirlanda ${ }^{1}$ \\ 1 INAF - Osservatorio Astronomico di Brera, Via Bianchi 46, 23807 Merate, Italy \\ e-mail: gabriele.ghisellini@brera.inaf.it \\ 2 Space Science Data Center - Agenzia Spaziale Italiana (SSDC-ASI), Via del Politecnico, s.n.c., 00133 Roma, Italy \\ 3 INAF-Osservatorio Astronomico di Roma, Via Frascati 33, 00078 Monteporzio Catone, Italy \\ ${ }^{4}$ Dipartimento di Fisica G. Occhialini, Univ. Milano-Bicocca, P.za della Scienza 3, 20126 Milano, Italy \\ 5 Scuola Internazionale Superiore di Studi Avanzati, Via Bonomea 265, 34135 Trieste, Italy \\ ${ }^{6}$ Kavli Institute for Particle Astrophysics and Cosmology, SLAC National Accelerator Laboratory, Menlo Park, CA 94025, USA
}

Received 22 April 2019 / Accepted 1 June 2019

\section{ABSTRACT}

\begin{abstract}
We observed three blazars at $z>2$ with the NuSTAR satellite. These were detected in the $\gamma$-rays by Fermi/LAT and in the soft X-rays, but have not yet been observed above $10 \mathrm{keV}$. The flux and slope of their X-ray continuum, together with Fermi/LAT data allows us to estimate their total electromagnetic output and peak frequency. For some of them we were able to study the source in different states, and investigate the main cause of the different observed spectral energy distribution. We then collected all blazars at redshifts greater than 2 observed by NUSTAR, and confirm that these hard and luminous X-ray blazars are among the most powerful persistent sources in the Universe. We confirm the relation between the jet power and the disk luminosity, extending it at the high-energy end.
\end{abstract}

Key words. radiation mechanisms: non-thermal - relativistic processes $-\gamma$ rays: general $-\mathrm{X}$-rays: general

\section{Introduction}

Blazars are radio-loud AGNs whose relativistic jet points directly at us, that is, with a viewing angle $\theta_{\mathrm{v}} \lesssim 1 / \Gamma$ with respect to the jet axis, where $\Gamma$ is the jet bulk Lorentz factor. The jet emission is greatly boosted by relativistic beaming, making blazars highly visible even at high cosmic distances.

The beamed nonthermal spectral energy distribution (SED) of powerful blazars is characterized by two broad distinctive humps. Most of the electromagnetic output of very powerful blazars is in the megaelectronvolt band, in the exact position for which there is no sensitive instrument to make observations. We can detect blazars however in the adjacent bands, through Fermi/LAT ( $>100 \mathrm{MeV})$ or in the hard X-rays, through INTEGRAL, Swift/BAT and NuSTAR. Only NuSTAR has the spectral resolution (through pointed observations) to accurately find, together with the LAT data (detections and upper limits), the peak frequency and luminosity of the blazar emission. We claimed (Ghisellini et al. 2010, hereafter G10) that the trend of lower intrinsic peak frequency with larger luminosity observed in blazars of low and intermediate power continues to be valid also at the extremely high-power end of the population. This was based on blazars detected by BAT, but not by LAT. Instead, considering blazars detected by both instruments, Ajello et al. (2009) claimed that no trend was visible. In addition to this controversial intrinsic property, the K-correction nevertheless favors the detection in the hard X-ray band of blazars at high redshifts. Therefore, the most powerful persistent objects of the Universe should be found in the hard X-ray band. Looking for these extreme objects, we proposed to use NUSTAR to observe a few blazars at $z>2$ that have already been detected by Fermi/LAT, but not by Swift/BAT, hoping to shed light on the intrinsic properties of these sources, and in particular on the possible relation between the peak frequency of the high-energy component of the SED and its luminosity.
Another key question in modern cosmology pertains to how supermassive black holes (SMBH) gained most of their mass, especially at the highest redshifts probed by current observations. Most high- $z$ searches of SMBHs concern radio-quiet objects, but a very promising alternative approach concerns radio-loud ones, and specifically blazars. Beaming makes blazars a unique tool in assessing the number density of radio-loud SMBHs at high redshift. In fact, for any confirmed high-redshift blazar there must exist other $2 \Gamma^{2}=450(\Gamma / 15)^{2}$ sources sharing the same intrinsic properties, but whose jets are not pointing at us. Some SMBHs with masses in excess of $10^{9} M_{\odot}$ were already in place when the Universe was only $\simeq 700$ Myrs old (e.g., ULAS J1120+0641 at $z=7.08$, Mortlock et al. 2011; ULAS J1342+0928 at $z=7.5$, Bañados et al. 2018). Their very existence is difficult to reconcile with black hole growth at the Eddington rate starting from stellarsized seeds (e.g., Volonteri et al. 2010).

To the three blazars observed for the first time by NuSTAR, we have added all other blazars with $z>2$ observed by NUSTAR in order to better understand their common properties. We show that all of them belong to the group of the most powerful blazars both in their jet and in their accretion disk properties, fully confirming the fact that the jet power is proportional to the accretion luminosity and our expectations that the hard X-ray selection of high-redshift blazars picks up the most powerful sources.

We use a flat $\Lambda$ CDM cosmology with $h=\Omega_{\Lambda}=0.7$.

\section{Data analysis}

Table 1 lists the three blazars observed by NuSTAR, selected among all blazars at $z>2$ already detected by Fermi/LAT (Atwood et al. 2009) with a [0.3-10 keV] flux larger than $10^{-12} \mathrm{erg} \mathrm{cm}^{-2} \mathrm{~s}^{-1}$ and not already observed by NuSTAR, or by Swift/BAT. This table also reports the redshift, the flux at $5 \mathrm{GHz}$, 
Table 1. Selected targets.

\begin{tabular}{lccccccccc}
\hline \hline RA & Dec & Alias & $z$ & $\begin{array}{c}F_{5} \\
\mathrm{Jy}\end{array}$ & $\begin{array}{c}F_{[0.3-10]} \\
\mathrm{cgs}\end{array}$ & $\Gamma_{X}$ & $\begin{array}{c}L_{X} \\
\mathrm{erg} \mathrm{s}^{-1}\end{array}$ & $\begin{array}{c}m_{R} \\
M_{\mathrm{BH}}^{\mathrm{vir}} \\
M_{\odot}\end{array}$ \\
\hline 012642 & +255901 & PKS 0123+25 & 2.358 & 1.4 & $2.5 \mathrm{e}-12$ & 1.4 & $5.2 \mathrm{e} 46$ & 17.8 & $1.8 \mathrm{e} 9$ \\
022928 & -364356 & PKS 0227-369 & 2.115 & 0.4 & $1.3 \mathrm{e}-12$ & 1.4 & $2.2 \mathrm{e} 46$ & 19.0 & - \\
050112 & -015914 & TXS 0458-020 & 2.291 & 3.3 & $1.4 \mathrm{e}-12$ & 1.5 & $3.1 \mathrm{e} 46$ & 19.0 & $4.6 \mathrm{e} 8$ \\
\hline
\end{tabular}

Notes. The table lists: coordinates (J2000), alias, redshift $z$, radio flux at $5 \mathrm{GHz}$; X-ray flux in the $0.3-10 \mathrm{keV}$ band; X-ray photon spectral index; K-corrected $0.3-10 \mathrm{keV}$ luminosity; $R$ magnitude; virial black hole mass. The virial black hole masses are respectively from: Kelly \& Bechtold (2007), Shaw et al. (2012), Fan \& Cao (2004), Shen et al. (2011).

the optical magnitude in the $R$ band, and the estimate of the black hole mass obtained through the virial estimate, when available.

\subsection{NUSTAR}

The NUSTAR satellite (Harrison et al. 2013) observed PKS $0123+25$ on 2018 January 03 (obsID 60367001002), PKS 0227369 on 2017 August 10 (obsID 60367002002), and TXS 0458020 on 2018 April 26 (obsID 60367003001). The total net exposure times were 19.9, 23.3, and $20.7 \mathrm{ks}$, respectively.

The Focal Plane Modules A and B (FPMA and FPMB) data sets were processed with the NuSTARDAS software package (v.1.8.0) developed by the Space Science Data Center of the Italian Space Agency (SSDC-ASI, Italy) in collaboration with the California Institute of Technology (Caltech, USA). Calibrated and cleaned event files were produced with the nupipeline task using the version 20170705 of the NUSTAR Calibration Database (CALDB).

The three sources were all well detected above the background by the two NUSTAR hard X-ray telescopes up to $30 \mathrm{keV}$. The FPMA and FPMB energy spectra of the three sources were extracted from the cleaned and calibrated event files using a circular spatial region with a radius of 12 pixels ( $\sim 30$ arcseconds) centered on the target, while the background was extracted from nearby circular regions of 50 pixel radius. The ancillary response files were generated with the nuproducts task, applying corrections for the point spread function (PSF) losses, exposure maps, and telescope vignetting.

For all three observations the spectral analysis of the $N u S$ $T A R$ data was performed using the XSPEC package adopting a single power-law model with an absorption hydrogenequivalent column density fixed to the Galactic values given by Kalberla et al. $(2005)$, that is, $N_{\mathrm{H}}=6.8 \times 10^{20} \mathrm{~cm}^{-2}$ for PKS $0123+25, N_{\mathrm{H}}=2.4 \times 10^{20} \mathrm{~cm}^{-2}$ for PKS $0227-369$ and $N_{\mathrm{H}}=6.0 \times 10^{20} \mathrm{~cm}^{-2}$ for TXS 0458-02. All spectra were binned to ensure a minimum of 30 counts per bin and energy channels below $3.0 \mathrm{keV}$ and above $30.0 \mathrm{keV}$ were excluded. A multiplicative constant factor was included to take into account crosscalibration uncertainties between the two telescopes (NuSTAR FPMA and FPMB). We found that this model fitted the spectral data very well for all three sources in the considered energy band. The results of the spectral fits are given in Table 2 .

\subsection{Swift/XRT}

The Neil Gehrels Swift Observatory (Gehrels et al. 2004) observed PKS $0123+25$ with the X-ray Telescope (XRT, Burrows et al. 2005) simultaneously with NuSTAR, namely on 2018 January 03 and January 4 (obsIDs 00088100001, 00088100002), for a total net exposure time of $2.0 \mathrm{ks}$.
The XRT observations were carried out with the Photon Counting (PC) readout mode. The XRT data were first processed using the XRT Data Analysis Software (XRTDAS, v.3.4.1), which was developed by SSDC-ASI. Standard calibration and cleaning processing steps were applied using the xrtpipeline software module and using version 20180710 of the Swift/XRT Calibration Database (CALDB).

Source events for the spectral analysis were extracted in the $0.3-10 \mathrm{keV}$ energy band using a circular spatial extraction region with a radius of 20 pixels ( 47 arcseconds). The background was estimated using a nearby source-free circular region with a radius of 50 pixels. Corrections to the ancillary response files for PSF losses, CCD defects, and telescope vignetting were calculated and applied using the xrtmkarf software module.

For the spectral analysis the energy spectrum was grouped to ensure at least 20 counts in each bin. We adopted an emission model described by a single power law with an absorption hydrogen-equivalent column density fixed to the Galactic value of $N_{\mathrm{H}}=6.8 \times 10^{20} \mathrm{~cm}^{-2}$ (Kalberla et al. 2005). The results of the spectral fit were found to be consistent in slope and normalization with those derived from the NUSTAR observation, thus extending the observed spectral slope down to $0.3 \mathrm{keV}$, with a best-fit photon index of $\Gamma=1.7_{-0.3}^{+0.3}$.

For the two blazars PKS 0227-369 and TXS 0458-020 no simultaneous observations with $N u S T A R$ were carried out by the Neil Gehrels Swift Observatory.

\subsection{Fermi/LAT}

We analyzed the Fermi/LAT data around the NuSTAR pointings using the Pass- 8 data version and the public Fermi Science Tools version v11r5p3.

First we looked for nearly simultaneous data, with several choices of exposure time, until we derived a detection. The blazar TXS 0458-02 was in a bright state, and an integration time of just 2 days ( \pm 1 day around the NuSTAR pointing) was enough for a detection of $\sim 11 \sigma$. The other two objects instead require years of integration for a detection. We therefore considered two exposures, a short one of 30 days ( \pm 15 days around the $N u S T A R$ pointing) to derive a meaningful upper limit at the same epoch as NUSTAR, and a long one of years, in order to measure the average spectrum. The long exposure is 4 years for PKS 0123+25 (from May 24, 2014 to May 24, 2018) and 2 years for PKS 0227-369 (from May 24, 2016 to May 24). The results are reported in Table 3.

Gamma-ray events were selected from a region of interest (ROI) of $15^{\circ}$ using standard quality criteria, as recommended by the Fermi Science Support Center (FSSC). We performed the likelihood analysis in two steps. In the first step the XML model included all the sources in the preliminary LAT 8 year 
Table 2. Parameters of the X-ray spectral analysis of the NuSTAR data.

\begin{tabular}{lccccc}
\hline \hline \multicolumn{5}{c}{ PKS 0123+25 } \\
\hline Date & $\Gamma$ & $\begin{array}{c}F_{3-5 \mathrm{kev}} \\
\mathrm{erg} \mathrm{cm}^{-2} \mathrm{~s}^{-1}\end{array}$ & $\begin{array}{c}F_{5-10 \mathrm{kev}} \\
\mathrm{erg} \mathrm{cm}^{-2} \mathrm{~s}^{-1}\end{array}$ & $\begin{array}{c}F_{10-30 \mathrm{kev}} \\
\mathrm{erg} \mathrm{cm}^{-2} \mathrm{~s}^{-1}\end{array}$ & $\chi^{2} /$ d.o.f. \\
\hline 2018 Jan 03 & $1.68_{-0.12}^{+0.12}$ & $4.7 \times 10^{-13}$ & $7.7 \times 10^{-13}$ & $1.6 \times 10^{-12}$ & $13.3 / 30$ \\
\hline \multicolumn{5}{c}{ PKS 0227-369 } \\
\hline 2017 Aug 10 & $1.35_{-0.26}^{+0.27}$ & $9.6 \times 10^{-14}$ & $1.9 \times 10^{-13}$ & $5.5 \times 10^{-13}$ & $3.5 / 10$ \\
\hline \multicolumn{5}{c}{$\mathrm{TXS} \mathrm{0458-020}$} \\
\hline 2018 Apr 26 & $1.66_{-0.08}^{+0.08}$ & $8.3 \times 10^{-13}$ & $1.4 \times 10^{-12}$ & $3.0 \times 10^{-12}$ & $57.2 / 55$ \\
\hline
\end{tabular}

Notes. The errors are at $90 \%$ level of confidence for one parameter of interest. Fluxes are corrected for Galactic absorption.

Table 3. Parameters of the power-law fits to the Fermi/LAT data.

\begin{tabular}{lcccc}
\hline \hline $\begin{array}{l}\text { Source } \\
{[1]}\end{array}$ & TS & $\begin{array}{c}\text { Flux } \\
{[2]}\end{array}$ & $\begin{array}{c}\Gamma_{\text {LAT }} \\
{[3]}\end{array}$ & $\begin{array}{c}\text { Exp. } \\
{[5]}\end{array}$ \\
\hline PKS 0123+25 & 0.0 & $<2.18$ & 2.8 & $30 \mathrm{~d}$ \\
& 32.7 & $1.28 \pm 0.35$ & $2.89 \pm 0.23$ & $4 \mathrm{y}$ \\
PKS 0227-369 & 0.0 & $<1.38$ & 29.7 & $30 \mathrm{~d}$ \\
& 63.3 & $1.61 \pm 0.29$ & $2.73 \pm 0.14$ & $2 \mathrm{y}$ \\
TXS 0458-02 & 137.8 & $46.2 \pm 8.2$ & $2.30 \pm 0.14$ & $2 \mathrm{~d}$ \\
\hline
\end{tabular}

Notes. Column [1]: object name; Col. [2]: test statistics (Mattox et al. 1996); Col. [3]: integrated photon flux or 95\% upper limit in the 0.1-300 $\mathrm{GeV}$ band, in units of $10^{-8} \mathrm{~cm}^{-2} \mathrm{~s}^{-1}$; Col. [4]: photon index of the LAT spectrum, measured or assumed for the upper limit; Col. [5]: total LAT exposure, around the NUSTAR pointing, in days (d) or years (y).

point-source list (FL8Y). We then performed a second likelihood fit using the XML model from the first step, optimized by dropping all sources with a $T S<1$. The analysis was performed with the NEWMINUIT optimizer, using an unbinned likelihood for the short datasets and a binned likelihood for the long exposures, with bins of $0.1^{\circ}$. Furthermore, each decade of energy was split in 10 bins.

The LAT data points for the SED were obtained by binning the spectrum with two bins per decade in energy, in the $0.1-$ $100 \mathrm{GeV}$ range, and performing a likelihood analysis in each single energy bin. In the XML model all parameters were kept fixed to the best-fit values, except for the normalization of the target and of the two backgrounds (isotropic and Galactic). A binned or unbinned likelihood was used if the total number of counts in the bin was higher or lower than 15000 , respectively. A Bayesian upper limit was calculated if in that bin the target had a $T S<9$ or $n$ pred $<3$. The light curves were obtained by performing an unbinned likelihood analysis in each time bin of 7 days, leaving the parameters of the brightest or variable FL8Y sources in the ROI to vary freely within an $8^{\circ}$ radius of the target.

\section{Modeling}

We interpret the overall SEDs of our sources with a leptonic, one-zone jet emission model plus the contribution from an accretion disk, its X-ray corona, and a molecular torus, that is absorbing and re-emitting in the infrared a fraction of the disk radiation. The details of the model are given in Ghisellini \& Tavecchio (2009, 2015) and here we summarize its main features.

- The emitting region producing the non-thermal radiation is assumed to be spherical, with radius $R$ and at a distance $R_{\text {diss }}$ from the central black hole. The jet is assumed conical, with semi-aperture angle $\psi$. Although $\psi \Gamma \sim 1$ is borne out by numerical simulations of jet acceleration, jets could have a parabolic shape while accelerating, becoming conical when coasting (e.g., Marscher 1980; Komissarov et al. 2007). They could also recollimate at large distances, making the relation between the transverse radius $r$ and the distance $R_{\text {diss }}$ uncertain. We assume, for simplicity, $\psi=0.1$, corresponding to $5.7^{\circ}$ and $\psi \approx 1 / \Gamma$. The emitting plasma is assumed to move with a bulk motion of velocity $\beta c$ and Lorentz factor $\Gamma$ at a viewing angle $\theta_{\mathrm{v}}$ from the line of sight. The Doppler factor is $\delta=1 /\left[\Gamma\left(1-\beta \cos \theta_{\mathrm{v}}\right)\right]$.

- Throughout the emitting region relativistic electrons are continuously injected at a rate $Q(\gamma)\left[\mathrm{cm}^{-3} \mathrm{~s}^{-1}\right]$ for a time equal to the light-crossing time $R / c$. The shape of $Q(\gamma)$ is assumed to be a smoothly broken power law with a break at $\gamma_{\mathrm{b}}$ :

$Q(\gamma)=Q_{0} \frac{\left(\gamma / \gamma_{\mathrm{b}}\right)^{-s_{1}}}{1+\left(\gamma / \gamma_{\mathrm{b}}\right)^{-s_{1}+s_{2}}} \mathrm{~cm}^{-3}$

- The power injected in the form of relativistic electrons is

$P_{\text {inj }}^{\prime}=m_{\mathrm{e}} c^{2} \int Q(\gamma) \gamma \mathrm{d} \gamma$

This is calculated in the comoving frame. We solve the continuity equation to find the energy distribution $N(\gamma)\left[\mathrm{cm}^{-3}\right]$ of the emitting particles at the particular time $R / c$, when the injection process is assumed to end. We account for synchrotron and inverse Compton cooling and $e^{ \pm}$pair production and reprocessing; although in our sources, $e^{ \pm}$pairs are not important.

- The magnetic field $B$ is tangled and uniform throughout the emitting region.

- There are several sources of radiation external to the jet: 1. The broad line region (BLR) is assumed to re-emit $10 \%$ of the accretion luminosity from a shell-like distribution of clouds located at a distance $R_{\mathrm{BLR}}=10^{17} L_{\mathrm{d}, 45}^{1 / 2} \mathrm{~cm}$;

2 . The IR emission from a dusty torus, located at a distance $R_{\mathrm{IR}}=$ $2.5 \times 10^{18} L_{\mathrm{d}, 45}^{1 / 2} \mathrm{~cm}$

3. The direct emission from the accretion disk, including its X-ray corona;

4. The starlight contribution from the inner region of the host galaxy, and the cosmic background radiation.

All these contributions are evaluated in the blob comoving frame, where we calculate the corresponding inverse Compton radiation from all these contributions, and then transform this into the observer frame.

- The numerical code we use is not time dependent: it gives a "snapshot" of the predicted SED at the time $R / c$, when the particle distribution $N(\gamma)$ and consequently the produced flux are at their maximum. 
- For powerful sources, the radiative cooling is efficient and the cooling timescale can be shorter than $R / c$ even for lowenergy particles. This implies that $\gamma_{\text {peak }}$, the random Lorentz factor of the electron emitting most of the radiation, is close to $\gamma_{\mathrm{b}}$.

- The size of the emitting region is rather compact, as indicated by the short variability timescales observed in blazars. As a consequence, the synchrotron flux is self-absorbed at high frequencies, in the submillimeter band. Therefore the model cannot account for the radio emission at lower frequencies, which must be produced by more extended regions of the jet.

- To calculate the flux produced by the accretion disk, we adopt a standard Shakura \& Sunyaev (1973) disk (see Ghisellini \& Tavecchio 2009). This model depends mainly on the accretion rate (regulating the total disk luminosity) and on black hole mass (regulating the location of the peak of the emission). This allows us to also fit the thermal radiation seen in the optical-UV range, and to estimate the accretion rate and the black hole mass.

- The disk luminosity is independent of the adopted accretion model (e.g., standard Shakura \& Sunyaev, with zero spin, or an accretion disk around a Kerr black hole). Instead the estimate of the mass does depend on the assumed accretion model (see e.g., Calderone et al. 2013; see also Campitiello et al. 2018 who studied how the black hole spin and the special and general relativistic effects impact on the determination of the mass of the black hole).

- The total jet power is the sum of the power carried by particles (we assumed one cold proton per emitting electron), magnetic field, and radiation. Therefore, the estimate of the magnetic and particle power is model dependent because the particle number and the value of the magnetic field depend on which model we are using to interpret the data (leptonic or hadronic, molti or one-zone, and so on). This is calculated at the dissipation region, through

$P_{\mathrm{i}}=\pi \psi^{2} R_{\mathrm{diss}}^{2} U_{\mathrm{i}} \Gamma^{2} \beta c$,

where the subscript "i" can stand for protons, electrons, magnetic field, or radiation, and $U$ is the corresponding energy density, as calculated in the comoving frame. The power in radiation is instead model independent. It can be calculated with the equation above, that can be re-written as (for viewing angles $\left.\theta_{\mathrm{v}} \sim 1 / \Gamma\right)$ :

$P_{\mathrm{r}} \sim 2 \frac{L_{\text {jet }}^{\text {bol }}}{\Gamma^{2}}$,

where $L_{\text {jet }}^{\text {bol }}$ is the bolometric observed luminosity produced by the jet. This is an observable. Therefore, only the knowledge of $\Gamma$ enters this estimate. This makes $P_{\mathrm{r}}$ almost model independent. It is a lower limit of the jet power. $P_{\text {jet }}$ is the sum of the different components.

- The uniqueness of the parameter values was discussed in some detail in Ghisellini \& Tavecchio (2015). We stressed there that in the framework of our leptonic, one-zone model, it is possible to find a unique solution for fitting the SED, but only if the data are of sufficient quality. One would need simultaneous data from the millimeter to the $\gamma$-rays, and this is possible only in a few cases. We are therefore constrained to assume that the nonsimultaneous data we have collected are a reasonably good representation of the SED. We tried to constrain the $\gamma$-ray flux and slope as best we could by analyzing the Fermi/LAT data as close as possible to the NUSTAR observations. In addition, when possible we compare the resulting SED with the SED corresponding to other states of the sources in search of the possible causes of variations.

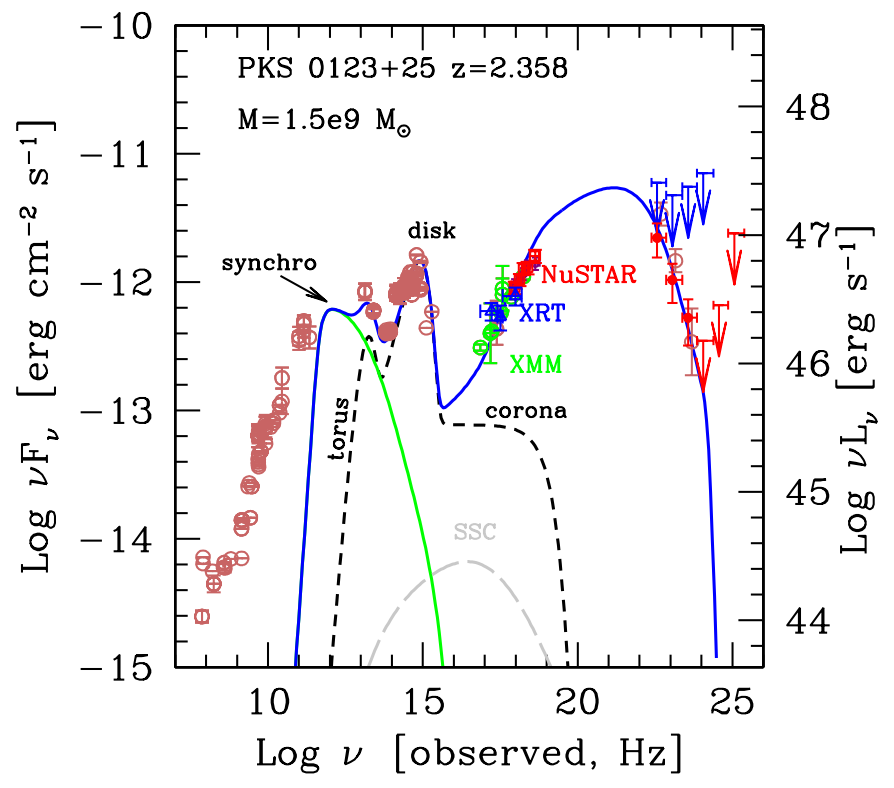

Fig. 1. Overall SEDs of PKS 0123+25. Besides our data (red points), we show the archival data collected from the ASI/SSDC database. We have indicated in green the XMM-Newton data, taken in January 2009, and in blue the Swift/XRT data taken simultaneously with the NuSTAR observation. The blue arrows in the $\gamma$-ray band are upper limits obtained integrating over 30 days ( 15 days before and 15 days after the NuSTAR observation). Red $\gamma$-ray points and arrows correspond to the average flux during the last 4 years. The lines are the result of the modeling (see text).

\section{Results}

We show the overall SEDs of the three blazars analyzed in this paper in Figs. 1, 4, and 6. The SEDs of PKS 0123+25 and PKS 0227-369 show the presence of a thermal component at opticalUV frequencies, that we interpret as being due to a standard accretion disk. Perhaps more surprising, this thermal emission is not clearly visible in TXS 0458-02, most probably because it is hidden by the dominating synchrotron spectrum. Besides showing our data, the figures report the archival data from the ASI/SSDC database ${ }^{1}$.

\subsection{PKS $0123+25$}

The NUSTAR data of this source lie on the extrapolation of the lower-energy X-ray data taken by XMM-Newton January 8 , 2009, and the Swift/XRT data taken simultaneously with $N u S$ $T A R$. Integrating the Fermi/LAT data 15 days before and 15 day after the NUSTAR observation, the source was not detected. The corresponding 95\% upper limits are shown in Fig. 1 together with the Fermi/LAT spectrum integrating over the last 4 years. The upper limits are consistent with the spectrum obtained with the long exposure, indicating no flares during the NUSTAR observation.

The optical spectrum can be well fitted by a standard accretion-disk model, and we find a black hole mass of $M=$ $1.5 \times 10^{9} M_{\odot}$ and a disk luminosity of $L_{\mathrm{d}}=5.85 \times 10^{46} \mathrm{erg} \mathrm{s}^{-1}$, corresponding to $30 \%$ of the Eddington luminosity. This value agrees with the observed broad-line luminosities, as observed by the SDSS spectrum (DR13). We used the template of Francis et al. (1991) and assumed that $L_{\mathrm{d}}=10 L_{\mathrm{BLR}}$. In this way

\footnotetext{
1 https://tools.ssdc.asi.it/
} 


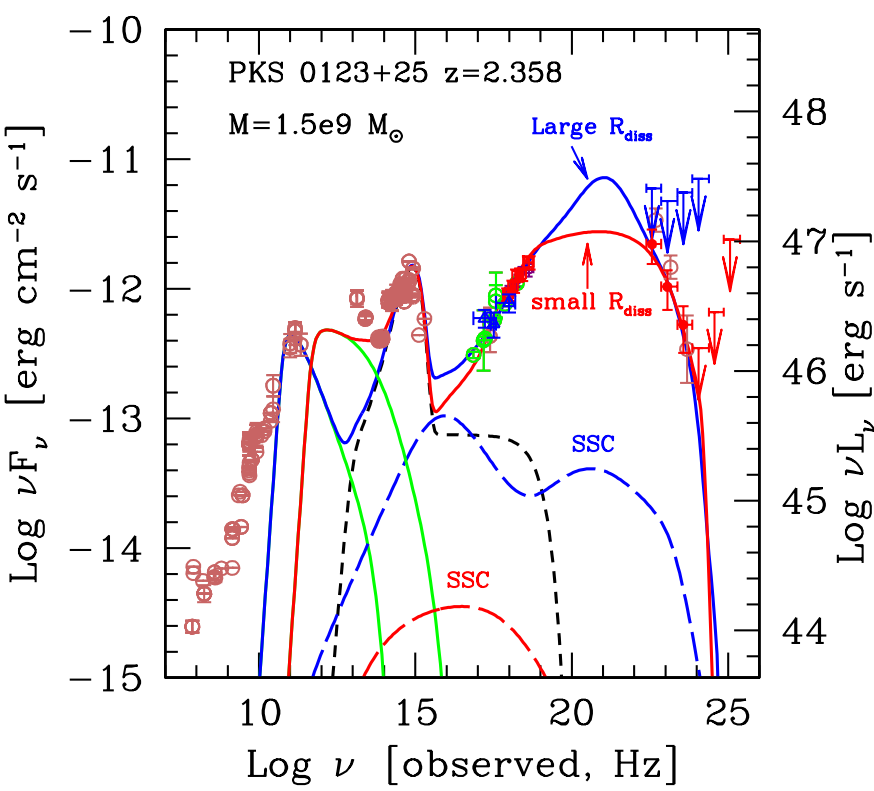

Fig. 2. Lines resulting from the modeling assuming that there is no torus, and assuming both a small $R_{\text {diss }}\left(=2.25 \times 10^{17} \mathrm{~cm}\right)$ and a large $R_{\text {diss }}\left(=3.6 \times 10^{18} \mathrm{~cm}\right)$. Parameters are listed in Table 4 . If we fit the highenergy emission, the model underproduces the near-IR flux. The long dashed line corresponds to the first- and the second-order Compton SSC contributions.

we derived $L_{\mathrm{BLR}}=10^{46} \mathrm{erg} \mathrm{s}^{-1}$ (using the CIV line); $L_{\mathrm{BLR}}=$ $7.3 \times 10^{45} \mathrm{erg} \mathrm{s}^{-1}$ (CIII] line) and $L_{\mathrm{BLR}}=1.6 \times 10^{45} \mathrm{erg} \mathrm{s}^{-1}(\mathrm{MgII}$ line). The contribution of both the torus and the jet emission can be found in the infrared band. In order to disentangle the two, we have assumed that the time-averaged $\gamma$-ray spectrum is indicative of the high-energy emission during the NUSTAR observation. In Fig. 2 we show the model SED assuming there is no torus: if we fit the high-energy SED, we under-reproduce the near-IR. We therefore assume that the near-IR flux is produced by the torus, and this helps us to find the peak of the high-energy SED and its dominance with respect to the synchrotron component. This information helps to constrain the magnetic field and $\gamma_{\text {peak }}$ allowing us to find a robust solution for the model parameters (assuming that the archival data are indicative of the real SED). Figure 3 compares the models assuming two different values for the aperture angle of the jet: $\psi=0.1=5.7^{\circ}$ (blue lines) and $\psi=0.023=1.3^{\circ}$ (red lines). The latter value corresponds to the average value of Fermi/LAT blazars derived by Pushkarev et al. (2017). Both models represent the data well, and are indistinguishable. The model with the smaller $\psi$ requires a larger $R_{\text {diss }}$ (factor 3) and a larger jet power (factor 3). For homogeneity with the blazars fitted previously, in the rest of the paper we use $\psi=0.1$. The parameters are listed in Table 4 .

\subsection{PKS 0227-369}

The X-ray flux was significantly lower during the NUSTAR observations with respect to an earlier Swift/XRT observation carried out in November 2008 (Ghisellini et al. 2009a,b). The shown $\gamma$ ray data (red symbols) refer to the last 2 years, and indicate a low state both with respect to the archival data and to an older flaring state. The slopes of both the X-ray and the $\gamma$-ray data are instead the same as the ones derived by the archival data. Unfortunately, during the NUSTAR observations, the source was not observed by Swift, meaning that we cannot check if any change occurred also in the optical-UV bands. However, we do not expect any

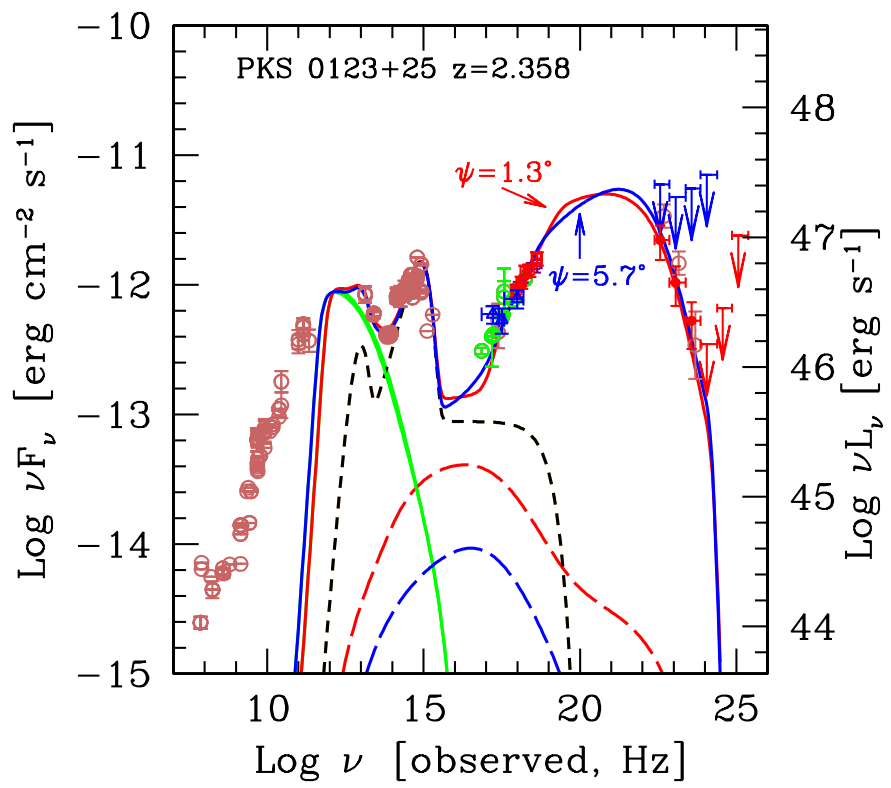

Fig. 3. Comparison between the best models assuming $\psi=0.1=5.7^{\circ}$ and $\psi=0.023=1.3^{\circ}$, as labeled. The long-dashed lines are the SSC contribution. Parameters are listed in Table 4.

strong flux variability in these bands, since they are produced by the accretion disk, whose emission is usually much more stable than that of the jet. Applying our standard disk model we derive $M=2 \times 10^{9} M_{\odot}$ and $L_{\mathrm{d}}=1.8 \times 10^{46} \mathrm{erg} \mathrm{s}^{-1}$, corresponding to $7 \%$ of the Eddington luminosity. We did not find any published optical spectra reporting the luminosity of the broad lines. However, the disk emission is clearly visible in this source and the accretion disk luminosity we found is therefore reliable. As in PKS $0123+25$, the IR flux is dominated by the jet synchrotron emission. As a consequence, the torus component is somewhat uncertain: in Fig. 4 we show a torus reprocessing half of the disk luminosity.

To model the source, we assumed that the radio-to-optical archival data give a good representation of the SED in this frequency range, and we tried to explain the change of the SED by changing the minimum number of parameters.

We find that the observed variability can be explained by changing the power of the relativistic electrons injected throughout the source that are responsible for the emission. The models shown differ by a factor of four in $P_{\text {inj }}^{\prime}$. Furthermore, the lower NuSTAR state is characterized by a slightly larger dissipation region, with a slightly smaller magnetic field and a larger value of the energy of the electrons emitting at the peaks of the SED. The total jet power is a factor three smaller than in the high state.

\subsection{TXS $0458-020$}

Figure 5 reports the Fermi/LAT light curve of the last 3 years, in order to show the variable behavior of this source. The dashed vertical line indicates the day of the NUSTAR observation.

Figure 6 shows the overall SED of the source, which is characterized by a relatively harder $\gamma$-ray spectrum with respect to the other two sources, as suggested by the nearly simultaneous Fermi/LAT data (red points). In this case the flux was high enough to allow the detection and some spectral determination integrating for one week around the NUSTAR observation.

Since the synchrotron jet emission hides the accretion disk component, we cannot directly fit the disk. We can derive a rough 
Table 4. Parameters for the models shown in Figs. 1, 2, 3, 4, and 6.

\begin{tabular}{|c|c|c|c|c|c|c|c|c|}
\hline Source & $z$ & $\begin{array}{c}M_{\mathrm{BH}}^{\mathrm{SED}} \\
M_{\odot} \\
\end{array}$ & $\begin{array}{c}L_{\mathrm{d}} \\
10^{45} \mathrm{erg} \mathrm{s}^{-1}\end{array}$ & $L_{\mathrm{T}} / L_{\mathrm{d}}$ & $\begin{array}{c}R_{\text {diss }} \\
10^{15} \mathrm{~cm}\end{array}$ & $\begin{array}{c}R_{\mathrm{BLR}} \\
10^{15} \mathrm{~cm}\end{array}$ & $\begin{array}{c}R_{\mathrm{T}} \\
10^{15} \mathrm{~cm} \\
\end{array}$ & $\begin{array}{c}P_{\text {inj }}^{\prime} \\
10^{45} \mathrm{erg} \mathrm{s}^{-1} \\
\end{array}$ \\
\hline PKS $0123+25$ & 2.358 & $1.5 \mathrm{e} 9$ & 58.5 & 0.3 & 270 & 764 & $3.1 \mathrm{e} 4$ & 0.017 \\
\hline PKS $0123+25$ no torus & 2.358 & $1.5 \mathrm{e} 9$ & 58.5 & 0 & 225 & 764 & - & 0.012 \\
\hline PKS $0123+25$ no torus & 2.358 & $1.5 \mathrm{e} 9$ & 58.5 & 0 & $3.6 \mathrm{e} 3$ & 764 & - & 0.3 \\
\hline PKS $0123+25\left(\psi=1.3^{\circ}\right)$ & 2.358 & $1.5 \mathrm{e} 9$ & 58.5 & 0.3 & 540 & 764 & $3.1 \mathrm{e} 4$ & 0.025 \\
\hline PKS 0227 - 369 new & 2.115 & $2 \mathrm{e} 9$ & 18.2 & 0.5 & 660 & 427 & $5.6 \mathrm{e} 3$ & 0.011 \\
\hline PKS 0227 - 369 old & 2.115 & $2 \mathrm{e} 9$ & 18.2 & 0.5 & 480 & 427 & $5.6 \mathrm{e} 3$ & 0.045 \\
\hline TXS $0458-020$ new & 2.291 & $8 \mathrm{e} 8$ & 10.4 & 0.5 & 144 & 322 & $2.7 \mathrm{e} 3$ & 0.11 \\
\hline TXS 0458 - 020 quiesc. & 2.291 & $8 \mathrm{e} 8$ & 10.4 & 0.5 & 132 & 322 & $2.7 \mathrm{e} 3$ & 0.025 \\
\hline TXS 0458 - 020 "flare" & 2.291 & $8 \mathrm{e} 8$ & 10.4 & 0.5 & 192 & 322 & $2.7 \mathrm{e} 3$ & 0.35 \\
\hline TXS $0458-020(\Gamma=7)$ & 2.291 & $8 \mathrm{e} 8$ & 10.4 & 0.5 & 192 & 322 & $2.7 \mathrm{e} 3$ & 0.25 \\
\hline
\end{tabular}

Notes. For the BLR we always assumed $L_{\mathrm{BLR}}=0.1 L_{\mathrm{d}}$. For all models we assumed $\psi=0.1=5.7^{\circ}$, unless otherwise noted. For a simple geometry (a spherical torus surrounding the disk), the ratio $L_{\mathrm{T}} / L_{\mathrm{d}}$ corresponds to the aperture angle $\theta_{\mathrm{T}}$ of the torus (the angle between the normal to the disk and the border of the torus): $L_{\mathrm{T}} / L_{\mathrm{d}}=\cos ^{2} \theta_{\mathrm{T}}$. A ratio $L_{\mathrm{T}} / L_{\mathrm{d}}=0.3$ gives $\theta_{\mathrm{T}}=57^{\circ}$, while $L_{\mathrm{T}} / L_{\mathrm{d}}=0.5$ gives $\theta_{\mathrm{T}}=45^{\circ}$.

Table 4. continued.

\begin{tabular}{lcccccccccc}
\hline \hline Source & $\begin{array}{c}B \\
\mathrm{G}\end{array}$ & $\Gamma$ & $\begin{array}{c}\theta_{\mathrm{v}} \\
\mathrm{deg}\end{array}$ & $\gamma_{\mathrm{b}}$ & $\gamma_{\max }$ & $s_{1}$ & $s_{2}$ & $\gamma_{\text {peak }}$ & $\log P_{\mathrm{r}}$ & $\log P_{\text {jet }}$ \\
\hline PKS 0123+25 & 6.0 & 11 & 3 & 400 & $5 \mathrm{e} 3$ & 1.5 & 4 & 98 & 45.6 & 47.2 \\
PKS 0123+25 no torus & 6.6 & 12 & 3 & $1 \mathrm{e} 3$ & $5 \mathrm{e} 3$ & 1.9 & 4.4 & 73 & 45.5 & 47.4 \\
PKS 0123+25 no torus & 0.036 & 22 & 2 & 200 & $5 \mathrm{e} 3$ & 1.9 & 4.4 & 181 & 45.5 & 49.5 \\
PKS 0123+25 $\left(\psi=1.3^{\circ}\right)$ & 6.7 & 11 & 3 & 550 & $5 \mathrm{e} 3$ & 1.9 & 4.3 & 54 & 45.6 & 47.7 \\
PKS 0227 - 369 new & 0.9 & 13 & 3 & 600 & $5 \mathrm{e} 3$ & 1 & 3.1 & 305 & 45.6 & 46.5 \\
PKS 0227 - 369 old & 1.3 & 13 & 3 & 250 & $5 \mathrm{e} 3$ & 0 & 3 & 181 & 46.3 & 46.9 \\
TXS 0458 - 020 new & 3.2 & 14 & 3 & 300 & $4 \mathrm{e} 3$ & -1 & 2.5 & 317 & 46.8 & 47.3 \\
TXS 0458 - 020 quiesc. & 8.1 & 13 & 3 & 190 & $3 \mathrm{e} 3$ & 0.7 & 3 & 116 & 46.0 & 47.1 \\
TXS 0458 - 020 “flare” & 2.5 & 18 & 3 & 200 & $4 \mathrm{e} 3$ & -1 & 3 & 170 & 47.5 & 48.2 \\
TXS 0458 - 020 ( $\Gamma=7)$ & 1.7 & 7 & 3 & 800 & $7 \mathrm{e} 3$ & -1 & 2.5 & 824 & 46.5 & 47.0 \\
\hline
\end{tabular}

Notes. Luminosities are in units of $\operatorname{erg~s}^{-1}$.

estimate of the accretion disk luminosity by the observation of the broad lines, which are seen in this source even if the continuum is dominated by the synchrotron emission. The CIV broad line has a flux $F_{\mathrm{CIV}}=2.6 \times 10^{-15} \mathrm{erg} \mathrm{cm}^{-2} \mathrm{~s}^{-1}$, corresponding to a luminosity of $L_{\mathrm{CIV}}=1.1 \times 10^{44} \mathrm{erg} \mathrm{s}^{-1}$. According to the template of Francis et al. (1991) this should correspond to a BLR total luminosity of $L_{\mathrm{BLR}}=9.7 \times 10^{44} \mathrm{erg} \mathrm{s}^{-1}$ and to a disk luminosity ten times larger: $L_{\mathrm{d}} \sim 10^{46} \mathrm{erg} \mathrm{s}^{-1}$.

For the black hole mass, we must consider that smaller masses, for a given $L_{\mathrm{d}}$, correspond to a disk spectrum peaking at larger frequencies. Therefore we can derive a lower limit to the black hole mass requiring that the disk emission does not overcontribute to the optical-UV flux. We obtain an upper limit to the mass, requiring that the disk that is emitting is geometrically thin and optically thick, and therefore has a luminosity larger than $0.01 L_{\text {Edd }}$. We chose $L_{\mathrm{d}}=0.1 L_{\text {Edd }}$ for $L_{\mathrm{d}} \sim 2 \times 10^{46} \mathrm{erg} \mathrm{s}^{-1}$, deriving $M=8 \times 10^{8} M_{\odot}$. These values are only indicative, and uncertain by at least a factor of two.

To explain the observed different states, we assumed that the archival data are representative of the quiescent state, while during the NUSTAR observation the source was in a high state. In March 2014 there was a Fermi/LAT flare almost brighter than in 2018, but unfortunately with no other observations at other frequencies. We show a possible fit for this flare, but only to illustrate the change of the parameters if the source were ever to resemble the proposed theoretical SED.
As usual, we look for a solution involving the smallest change of the minimum number of parameters to explain the observed variability. For the "NUSTAR state" the power injected in relativistic electrons is 4 times larger than in the quiescent state, but the magnetic field is $\sim 2.5$ times smaller. The slopes of the injected electron distribution are slightly harder and the total jet power in the NUSTAR state is twice as much as in quiescence. The "high" state would require more power in the injected electrons (more than ten times that in quiescence) and a still-smaller magnetic field, and the total jet power would be approximately 13 times larger. All these estimates are calculated assuming that the synchrotron part of the spectrum is well represented by the quiescent state, in turn shown by the archival data. This source was studied also in Ghisellini et al. (2011), where simultaneous Swift (UVOT and XRT) and Fermi/LAT observations are reported. They correspond to the black symbols in Fig. 6.

Recently, Lister et al. (2016) measured the apparent speed of a superluminal knot in this source, deriving an apparent speed $\beta_{\text {app }} \sim 6$. Although this is a lower limit to the value of the bulk Lorentz factor, and therefore consistent with the values used in Fig. 6, it is interesting to compare these models with the one using a smaller value of $\Gamma$. This is done in Fig. 7, which compares the models with $\Gamma=14$ and $\Gamma=7$, as labeled. The latter slightly underestimates the NUSTAR data, but can accurately reproduce the rest of the SED. The parameters listed in Table 4 indicate (for the $\Gamma=7$ case) that the jet power and the magnetic field are 


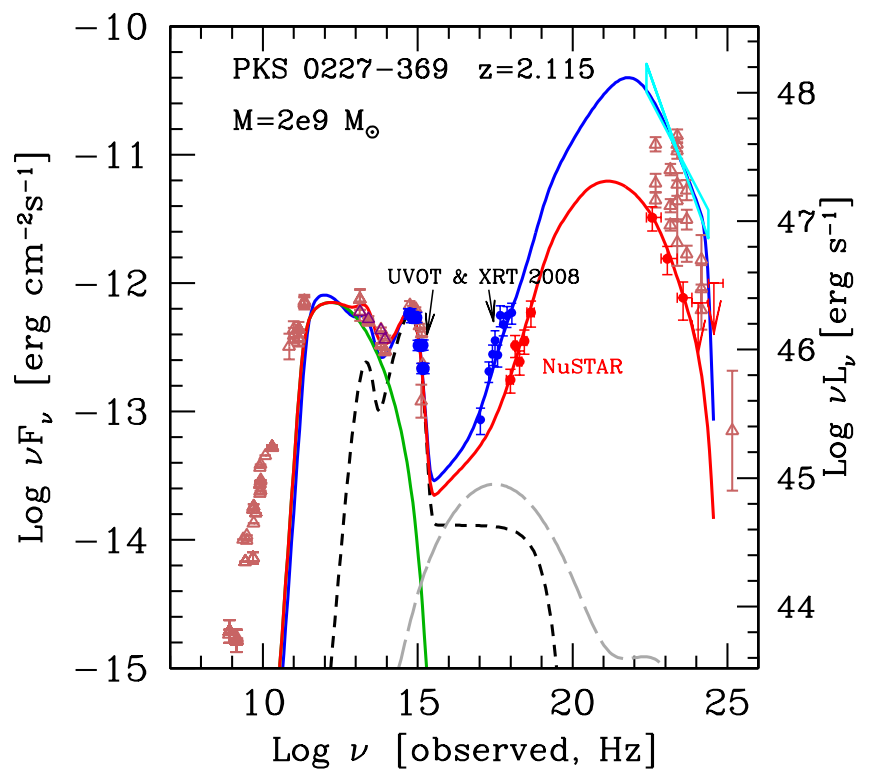

Fig. 4. Overall SEDs of PKS 0227-369. The X-ray flux was significantly lower during the NUSTAR observations with respect to an earlier Swift/XRT+UVOT observation carried out in November 2008. The red points in the Fermi/LAT band correspond to integrating the last two years of observations. This shows that the source was in a low state during this period of time.

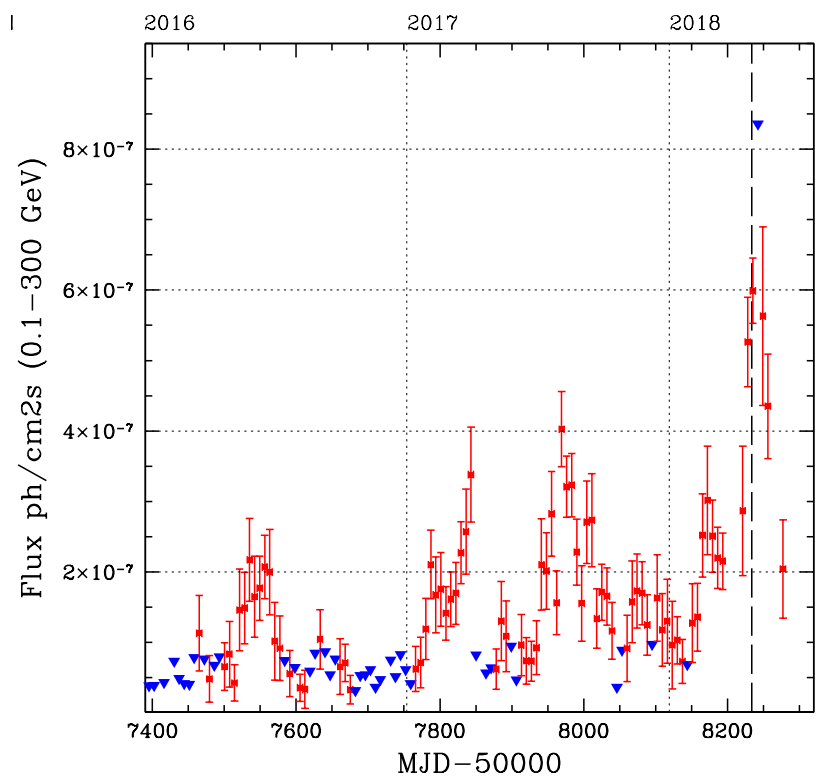

Fig. 5. $\gamma$-ray light curve of TXS 0458-02. Blue triangles are 95\% upper limits, calculated assuming a power law with photon spectral index $\Gamma=2$. The dashed vertical line corresponds to the NUSTAR observation epoch, when the source was in a very high $\gamma$-ray state.

slightly smaller, and the electron energies are larger. Overall, we note that the parameters are not vastly different.

\section{Discussion}

\subsection{Comparison with other $z>2$ NuSTAR blazars}

Table 5 reports the list of all blazars at $z>2$ observed by $N u S$ $T A R$; there are 11 sources. The table reports their redshift and the reference to the papers discussing the NUSTAR X-ray data. All 11 sources are FSRQs, and their SEDs are shown in Fig. 8, in the

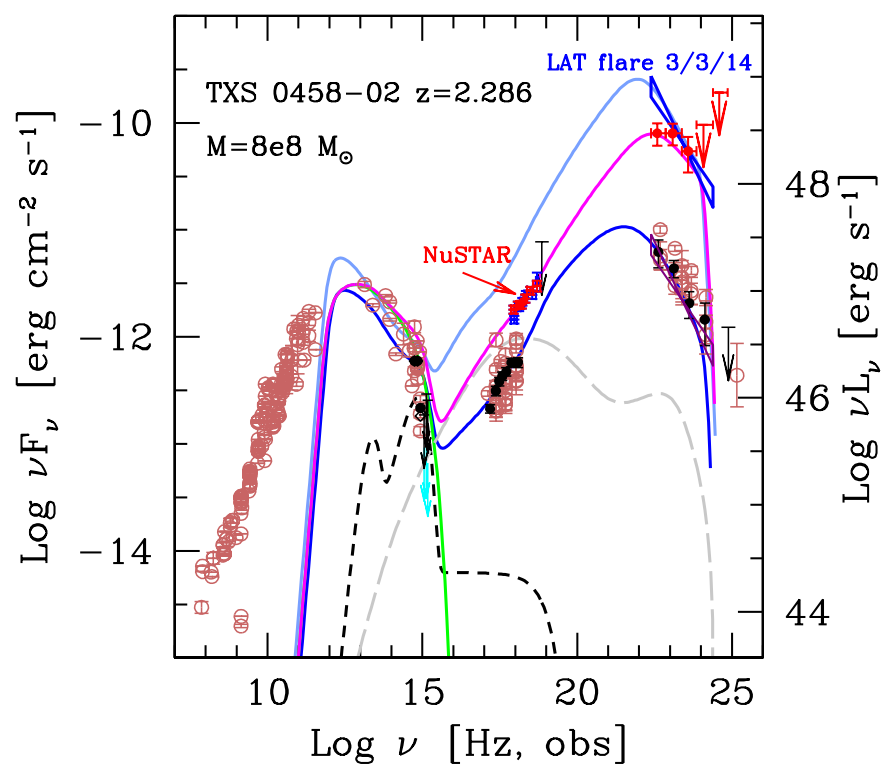

Fig. 6. Overall SEDs of TXS 0458-02, showing the changes in the high-energy emission due to its strong variability. Since unfortunately there are no low-frequency (mm-optical) data simultaneous to the varying high-energy flux, the models shown assume a quasi-constant flux at these frequencies. This illustrates how the model parameters would change in this case.

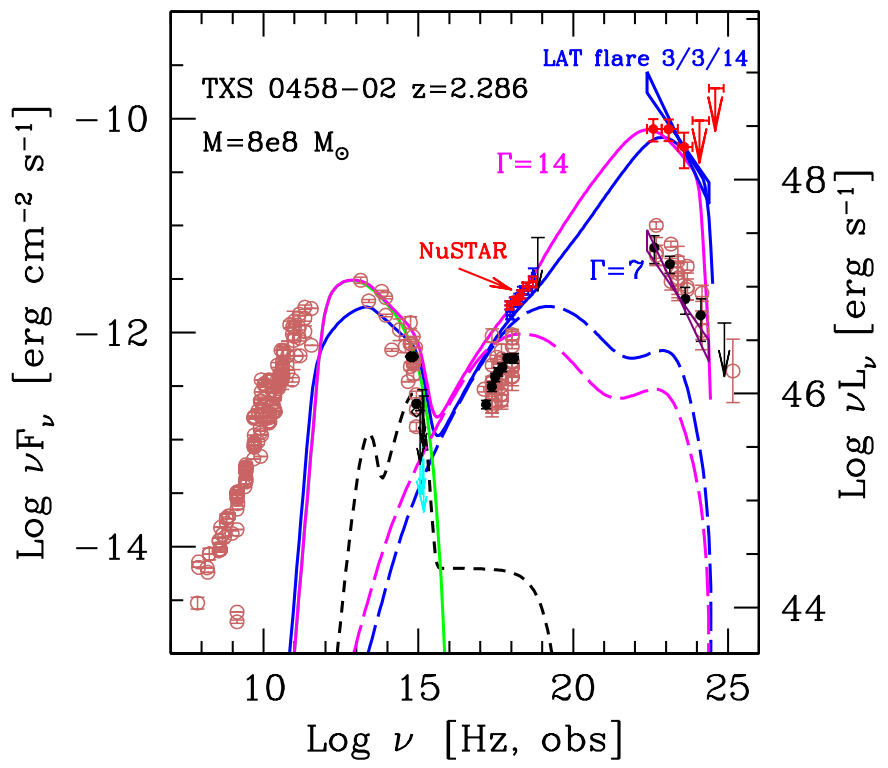

Fig. 7. Comparison of the models adopting $\Gamma=14$ and $\Gamma=7$, as labeled. Parameters are listed in Table 4 . The model with $\Gamma=7$ slightly underestimates the NUSTAR data.

$v L_{v}$ versus $v$ (rest frame) representation. In this way we can compare the rest frame SED of the sources. Most of the data come from archives (mostly ASI/SSDC) and the figure shows how similar the sources are in the radio-millimeter band, while they become different (and varying with a very large amplitude) at greater frequencies. We note the source S5 0014+813, the most luminous in the optical-UV, due to its extraordinary luminous accretion disk (Ghisellini et al. 2009a,b), and S5 0836+710, the most luminous in X-rays and $\gamma$-rays, where it reached a luminosity of $\sim 10^{50} \mathrm{erg} \mathrm{s}^{-1}$ during a flare observed on August 2, 2015 (Ciprini 2015). 
Table 5. Entire sample of $z>2$ blazars observed by NuSTAR

\begin{tabular}{lcc}
\hline \hline Name & $z$ & Ref. \\
\hline S5 0014+81 & 3.366 & S16, B18 \\
PKS 0123+25 & 2.358 & This paper \\
B0222+185 & 2.690 & S16, B18 \\
PKS 0227-369 & 2.115 & This paper \\
TXS 0322+222 & 2.066 & M17 \\
PKS 0446+11 & 2.15 & M17 \\
PKS 0451-28 & 2.564 & M17 \\
TXS 0458-020 & 2.291 & This paper \\
S5 0836+710 & 2.172 & T15, P15, B18 \\
B2 1023+25 & 5.3 & S13 \\
PKS 2149-306 & 2.345 & T15, D16, B18 \\
\hline
\end{tabular}

References. S16: Sbarrato et al. (2016); B18: Bhatta et al. (2018); S16: Sbarrato et al. (2016); M17: Marcotulli et al. (2017); T15: Tagliaferri et al. (2015); P15: Paliya (2015); S13: Sbarrato et al. (2013); D16: D'Ammando \& Orienti (2016).

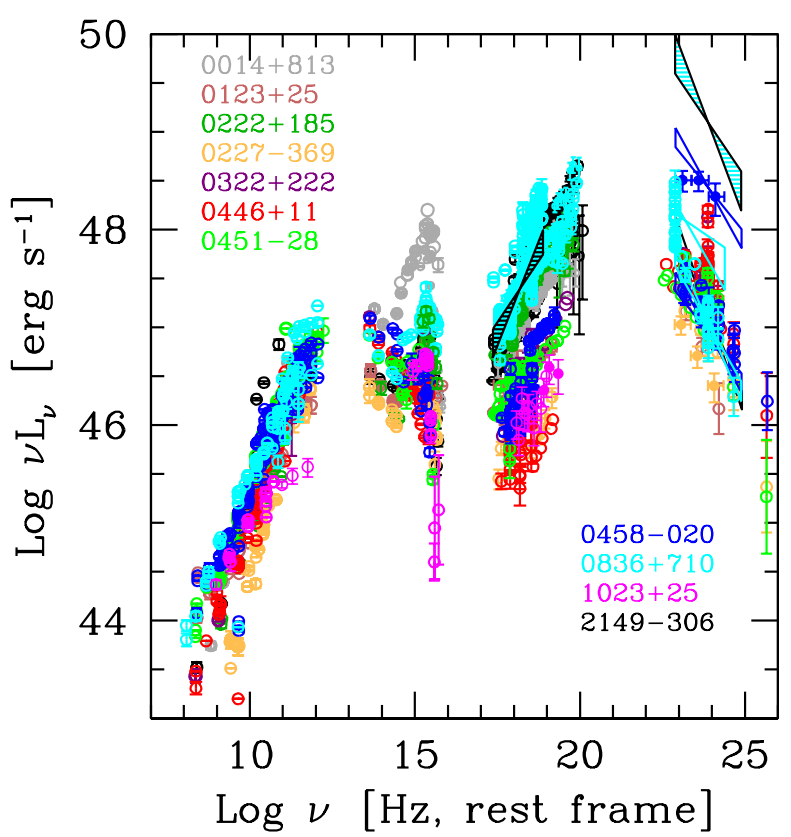

Fig. 8. SED of all 11 blazars at $z>2$ observed so far by NuSTAR. It can be noted that (1) the synchrotron hump is remarkably similar; (2) for several sources the accretion disk sticks out in the optical-UV band; (3) 0014+813 has an exceptionally powerful accretion disk; and (4) the $\mathrm{X}$-ray and $\gamma$-ray emission is more dispersed and variable.

The reason for the smaller dispersion of data points in the radio with respect to the other wavelengths is probably the lower amplitude variability in the radio band. Another reason for having less dispersion in the radio-millimeter band is that the Doppler amplification of the synchrotron flux scales as $F(v) \propto$ $\delta^{3+\alpha} \sim \delta^{3}$ (for flat spectral indices $\alpha=0$ ), while the amplification factor for the inverse Compton process, with photons produced externally to the jet, scales as $F(v) \propto \delta^{4+2 \alpha} \sim \delta^{5}$ (for X-ray spectral indices $\alpha \sim 0.5$ ) as pointed out by Dermer (1995) and illustrated in Fig. 5 of Ghisellini (2015).

We note that all sources show no sign of changing slope at the lowest radio frequencies, an indication that the jet emission is extremely strong and hides any contribution of the extended radio structure, which should have a steep (i.e., increasing at lower frequencies) spectrum. On the other hand, for almost all sources we do see the contribution of the accretion disk in the optical-

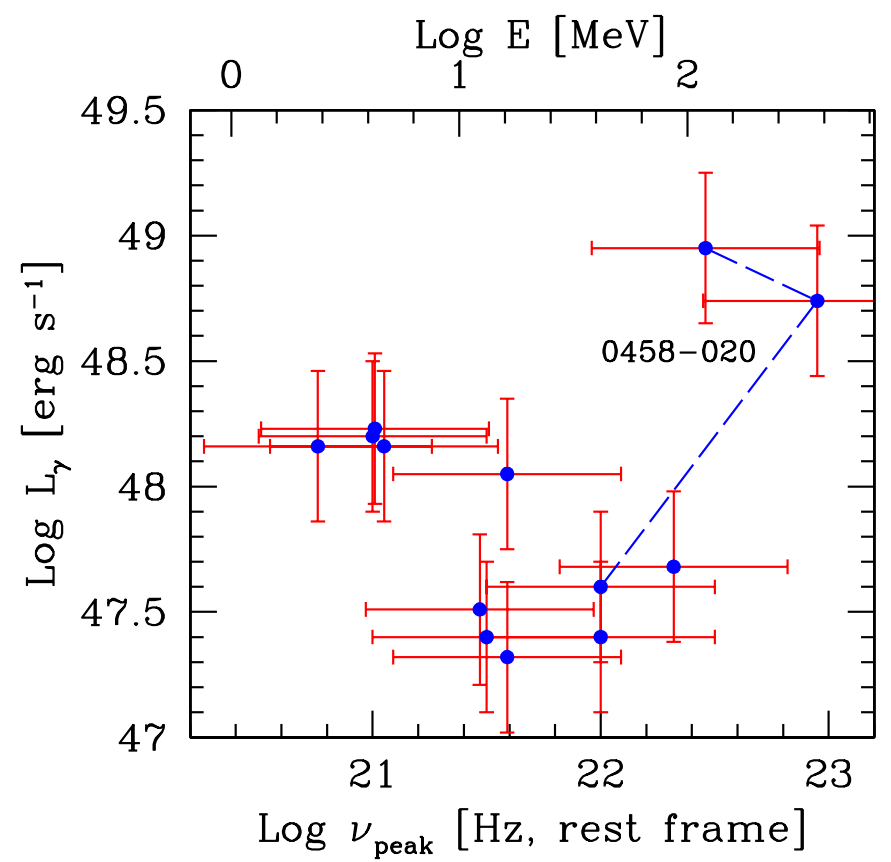

Fig. 9. Peak luminosity of the high-energy component as a function of its peak frequency. The dashed line connects three different states of TXS 0458-020. Error bars correspond to factor 3 uncertainties in $v_{\text {peak }}$ and factor 2 in $L_{\gamma}$. There is a weak trend of smaller luminosities for larger peak frequencies, with the exception of TXS 0458-020 when in the high state.

UV. The accompanying X-ray coronal emission is absent in these sources, completely overwhelmed by the beamed X-rays from the jet. As a consequence, there is no sign of the presence of the iron fluorescence line at $6.4 \mathrm{keV}$ (rest frame) for any of the sources.

The hardness of the X-ray spectrum coupled with the steepness of the $\gamma$-ray one indicates a spectral peak around $\sim 10 \mathrm{MeV}$. We can try to be more precise by extrapolating the X-ray and $\gamma$ ray spectra of each source and find out the matching frequency. The result is shown in Fig. 9: the $\gamma$-ray luminosity $L_{\gamma}$ is plotted against the peak frequency. For $L_{\gamma}$ we chose an average state, not the extreme flaring state. It should be borne in mind that this result can be affected by systematic errors, since the spectral shape around the peak is likely to be curved and not accurately described by a broken power law. The figure in any case suggests a trend (smaller $v_{\text {peak }}$ for larger $L_{\gamma}$ ) and an outlier (TXS 0458-020 in the high state).

\subsection{Seed photons from the BLR or the torus?}

The peak frequency $v_{\mathrm{C}}$ of the high-energy hump of blazars depends on the frequency of the seed photons, the energy of the relevant electrons contributing to the peak, the bulk Lorentz factor $\Gamma$, and the beaming factor $\delta$. For our sources, which are all very powerful, we can assume that $\delta \approx \Gamma$, implying that the viewing angle $\theta_{\mathrm{v}} \approx 1 / \Gamma$. If the emitting region is inside the broad-line region (i.e., $R_{\text {diss }}<R_{\mathrm{BLR}}$ ) the most important seed photons are the Ly $\alpha$ ones. Therefore, we expect

$v_{\mathrm{C}}=\frac{4}{3} \gamma_{\text {peak }}^{2} \nu_{\mathrm{Ly} \alpha} \frac{\Gamma^{2}}{1+z} ; \quad R_{\mathrm{diss}}<R_{\mathrm{BLR}}$

If $R_{\mathrm{BLR}}<R_{\mathrm{diss}}<R_{\text {torus }}$, the most important seed photons are the ones produced by the torus. These have a frequency related to the torus temperature, which has to be lower than $\sim 2000 \mathrm{~K}$ to avoid sublimation. 


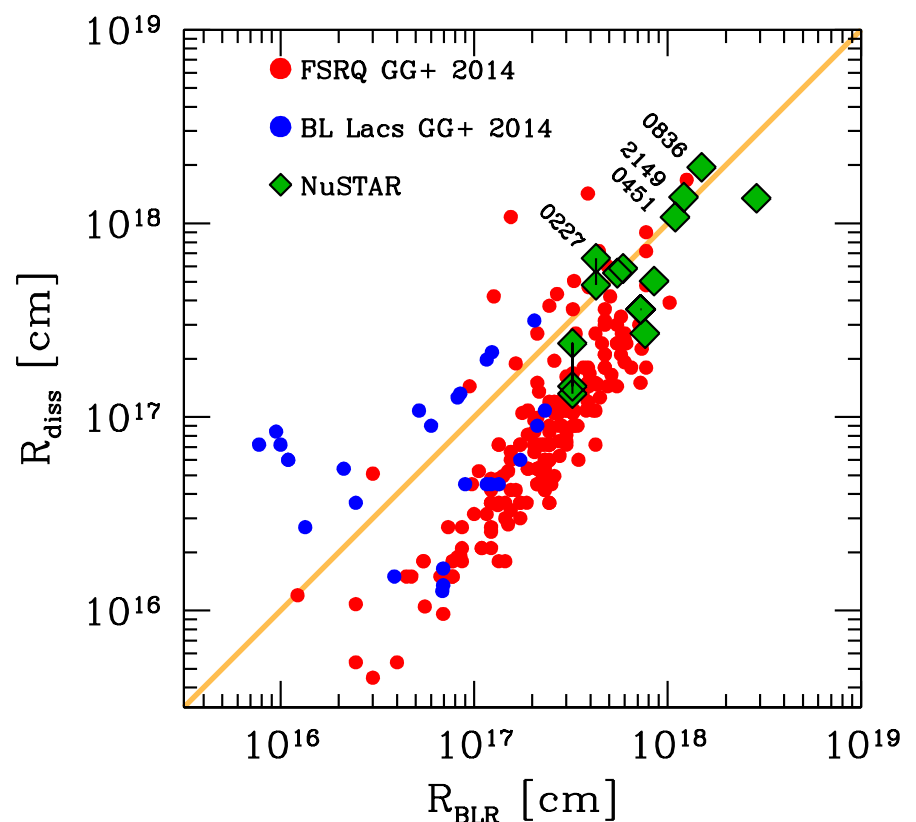

Fig. 10. Distance $R_{\text {diss }}$ at which most of the luminosity is produced as a function of the size of the broad-line region, $R_{\mathrm{BLR}}$. Blue ("BL Lacs", but with broad emission lines; see text) and red (FSRQ) data points are from Ghisellini et al. (2014). Green diamonds are our NuSTAR blazars. Different states of the same source are connected by a segment. For about $12 \%$ of all sources the dissipation region is located beyond the $\operatorname{BLR}\left(R_{\text {diss }}>R_{\mathrm{BLR}}\right)$.

$v_{\mathrm{C}}=\frac{4}{3} \gamma_{\text {peak }}^{2} v_{\text {torus }} \frac{\Gamma^{2}}{1+z} ; \quad R_{\mathrm{BLR}}<R_{\mathrm{diss}}<R_{\text {torus }}$

The ratio of the two $v_{\mathrm{C}}$ frequencies is $\sim 40\left(10^{3} \mathrm{~K} / T_{\text {torus }}\right)$. If the emitting region is at a distance that is greater than but close to $R_{\mathrm{BLR}}$, both types of seed photons are important, and we have an intermediate peak frequency as long as $\gamma_{\text {peak }}$ is the same. In general, one would expect that the radiative cooling time is affected by the nature of the seed photons: inside the BLR, the BLR radiation energy density is larger than that produced by the torus. Cooling is more severe, and this could favor smaller $\gamma_{\text {peak }}$. This compensates the larger seed photon energy. On the other hand, we calculate the particle distribution at the end of the injection, which lasts for a time $R / c$. We also assume that the jet is conical, and therefore $R \propto R_{\text {diss }}$ : if the emitting region is beyond $R_{\mathrm{BLR}}$, it is larger than if it is inside. This means that emission (and cooling) operate for a longer time, and this has the effect of decreasing $\gamma_{\text {peak }}$. Therefore, it is not obvious that sources dissipating beyond $R_{\mathrm{BLR}}$ should be "bluer" than the others. In any case, we have tried to see how many blazars studied previously by our group require $R_{\text {diss }}>R_{\mathrm{BLR}}$.

Figure 10 shows $R_{\mathrm{diss}}$ as a function of $R_{\mathrm{BLR}}$ for the sample of blazars studied in Ghisellini et al. (2014) and for the highredshift $N u S T A R$ FSRQs studied here. The figure shows that there is a small $(\sim 12 \%)$ fraction of sources with $R_{\text {diss }} \gtrsim R_{\mathrm{BLR}}$ and that there is an overall trend for $R_{\text {diss }}$ increasing more than linearly with $R_{\mathrm{BLR}}$. The $N U S T A R$ blazars require the largest $R_{\text {diss }}$ and $R_{\mathrm{BLR}}$ and nearly half of them dissipate beyond $R_{\mathrm{BLR}}$. We also consider the possibility that the $R_{\text {diss }} / R_{\mathrm{BLR}}$ ratio could be a function of the black hole mass. We do expect some dependence, because $R_{\mathrm{BLR}}$ depends on the black hole mass only through $L_{\mathrm{d}}$ (and we do expect a more luminous disk for larger black hole masses), while $R_{\text {diss }}$ should scale linearly with the mass if dissipation occurs at the same distance measured in units of the

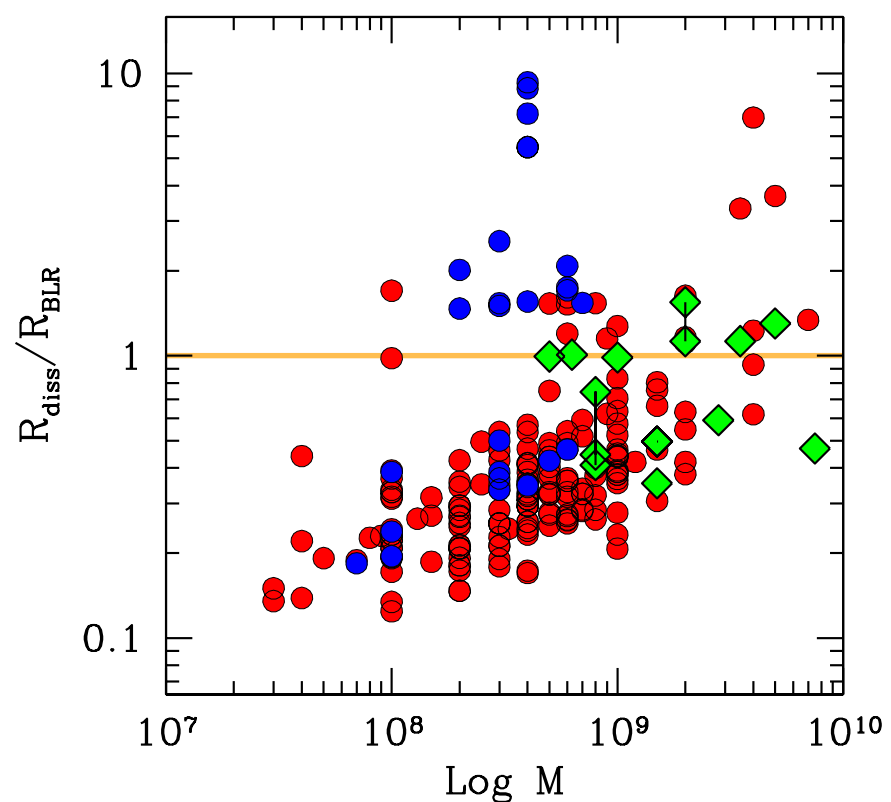

Fig. 11. Ratio $R_{\text {diss }} / R_{\mathrm{BLR}}$ as a function of the black hole mass. Blue ("BL Lacs") and red (FSRQ) from Ghisellini et al. (2014). Green diamonds are our NuSTAR blazars. Different states of the same source are connected by a segment.

Schwarzschild radius. Therefore we expect a dependence (albeit weak) for larger $R_{\text {diss }} / R_{\mathrm{BLR}}$ ratios for larger masses. Figure 11 shows this weak trend.

\subsection{The $\gamma_{\text {peak }}-U^{\prime}$ relation}

We now consider the relation between the electron random Lorentz factor $\gamma_{\text {peak }}$ of the electrons emitting at the peaks of the SED (both synchrotron and IC) and the magnetic plus radiation energy density in the comoving frame of the emitting region. This is shown in Fig. 12, which compares our high-z NuSTAR blazars with the samples of blazars studied by Celotti \& Ghisellini (2008) and Ghisellini et al. (2014). If considered altogether, there is a clear trend of decreasing $\gamma_{\text {peak }}$ for increasing energy density. On the other hand, the number of NUSTAR blazars is too small to derive any conclusions: they are, as are all the other powerful FSRQs, at the extreme of the distribution.

\subsection{Jet power and disk luminosity}

Finally, in Fig. 13, we consider the jet power as a function of the disk luminosity. The blue circles are labeled "BL Lacs", as was done by Ghisellini et al. (2014); they come from the sample of Sbarrato et al. (2013), containing 475 sources. Of these, Ghisellini et al. (2014) selected the few (26) objects with broad emission lines. Therefore, these "BL Lacs" should be considered as the low-disk-luminosity tail of the FSRQs. The relation between $P_{\text {jet }}$ and $L_{\mathrm{d}}$ remains significant even after accounting for the common dependence upon redshift, with a probability $P<10^{-8}$ of being random (Ghisellini et al. 2014). This figure clearly shows that the NUSTAR blazars studied in this paper are the most powerful. This remains true even if we consider the lower limit to the jet power given by $P_{\mathrm{r}}$, which is almost model independent. PKS $0836+710$ has the most powerful jet, and S5 $0014+81$ has the most powerful accretion disk. They extend the almost linear correlation between the two quantities found in Ghisellini et al. (2014), and confirm that 


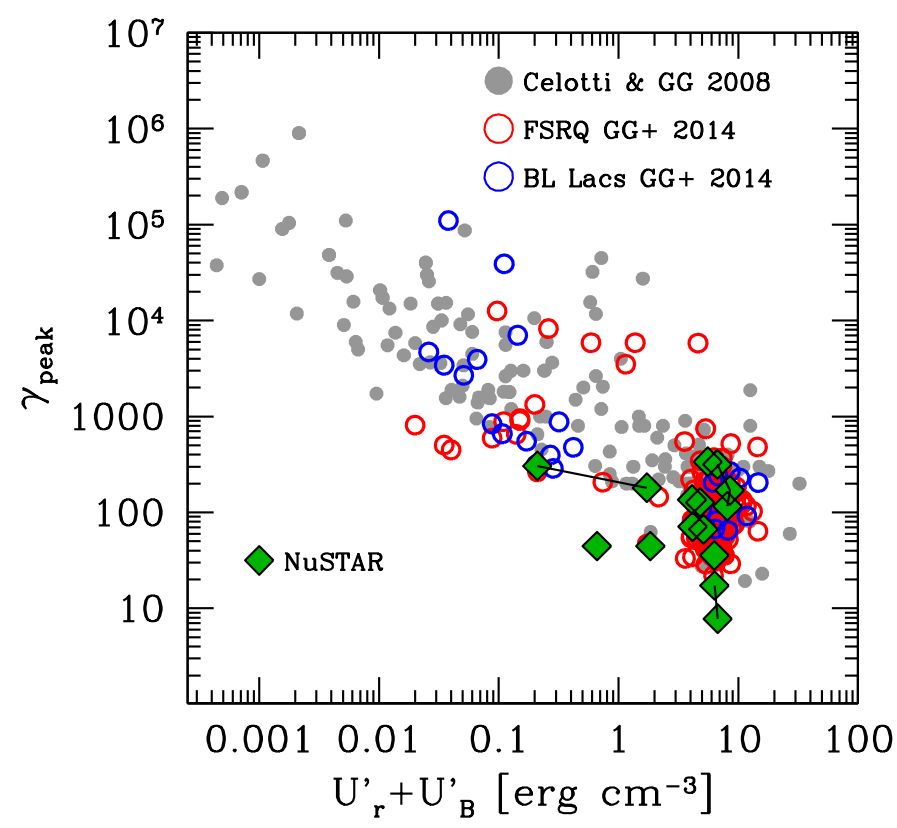

Fig. 12. Random Lorentz factor of the electrons emitting at the synchrotron and IC peaks vs the radiation+magnetic energy density as measured in the comoving frame. Grey filled circles: sources studied in Celotti \& Ghisellini (2008); empty red circles and blue circles: FSRQs and "BL Lacs" from Ghisellini et al. (2014); green diamonds: the sample of $z>2$ blazars observed by NUSTAR. Segments connect different states of the same source.

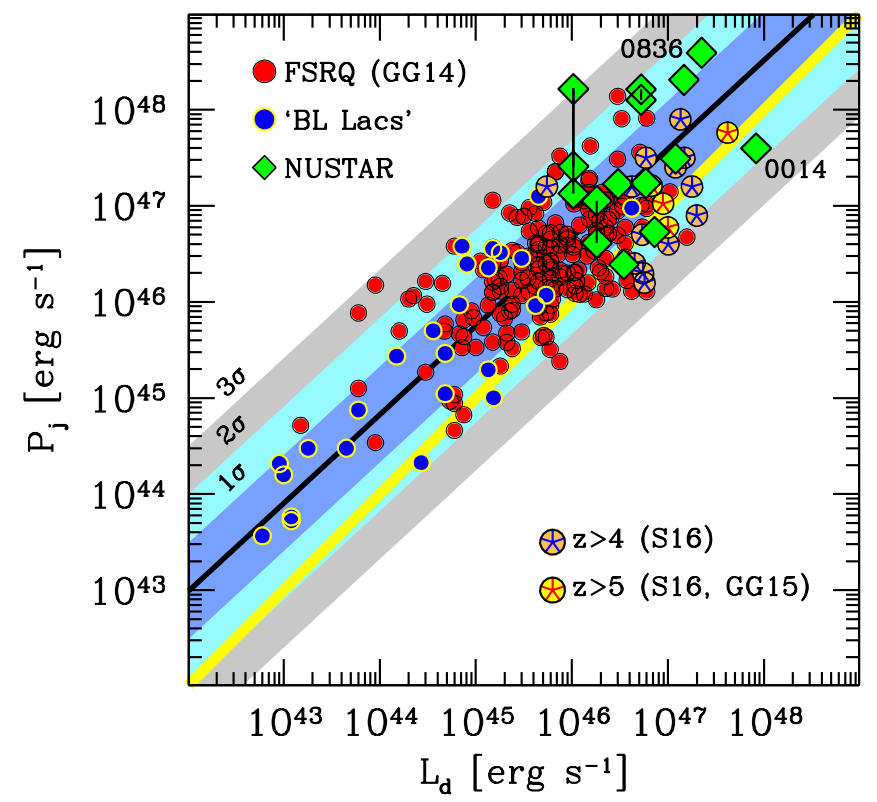

Fig. 13. Jet power as a function of disk luminosity of FSRQs (red) and "BL Lacs" (blue) considered in Ghisellini et al. (2014) compared with the NUSTAR blazars considered here. We also show the blazars with $z>4$ and $z>5$, considered in Sbarrato et al. (2016) and in Ghisellini et al. (2015). Segments connect different states of the same source. The NUSTAR blazars are among the most powerful, both in terms of their disk luminosity and jet power, with PKS $0836+710$ having the most powerful jet, and S5 0014+81 having the most powerful accretion disk. We note that the BL Lacs shown here were the only BL Lacs observed by Sbarrato et al. (2013) with broad emission lines. They must be considered the low disk luminosity tail of FSRQ.

active blazars have jets that are often more powerful than their accretion disks.

\section{Conclusions}

We report the results of our NUSTAR observations of three blazars at redshifts greater than 2 , and discuss the properties of all blazars at $z>2$ observed by NUSTAR and whose data are public. These objects form a sample of 11 sources. The main conclusions of our study are:

- Selection in the hard X-rays allows one to find the most powerful blazar jets and the most luminous accretion disks.

- PKS 0227-369 and TXS 0458-020 show significant variability in hard X-rays with respect to previous observations. This variability can be explained mainly by a change of power of the injected electrons and in part by a change of the magnetic field.

- All the high-z NuSTAR blazars observed so far belong to the class of very powerful FSRQs and have large black hole masses and accretion disks emitting well above the $0.01 L_{\mathrm{Edd}}$ rate.

- The high- $z$ NuSTAR blazars extend and confirm the relation between jet power and accretion-disk luminosity.

Acknowledgements. We acknowledge the ASI-NuSTAR grant ASI 1.05.04.95 and the grant ASI-INAF n. 2017-14-H.0 for funding.

\section{References}

Ajello, M., Costamante, L., Sambruna, R. M., et al. 2009, ApJ, 699, 603 Atwood, W. B., Abdo, A. A., Ackermann, M., et al. 2009, ApJ, 697, 1071 Bañados, E., Venemans, B. P., Mazzucchelli, C., et al. 2018, Nature, 553, 473 Bhatta, G., Mohorian, M., \& Bilinsky, I. 2018, A\&A, 619, A93

Burrows, D., Hill, J., Nousek, J., et al. 2005, Space Sci. Rev., 120, 165 Calderone, G., Ghisellini, G., Colpi, M., \& Dotti, M. 2013, MNRAS, 431, 210 Campitiello, S., Ghisellini, G., Sbarrato, T., \& Calderone, G. 2018, A\&A, 612, A59

Celotti, A., \& Ghisellini, G. 2008, MNRAS, 385, 283

Ciprini, S. 2015, ATel, 7870

D’Ammando, F., \& Orienti, M. 2016, MNRAS, 455, 1881

Dermer, C. D. 1995, ApJ, 360, 197

Fan, Z. H., \& Cao, X. 2004, ApJ, 602, 103

Francis, P. J., Hwett, P. C., Foltz, C. B., et al. 1991, ApJ, 373, 465

Gehrels, N., Chincarini, G., Giommi, P., et al. 2004, ApJ, 611, 1005

Ghisellini, G. 2015, JHEAp, 7, 163

Ghisellini, G., \& Tavecchio, F. 2009, MNRAS, 397, 985

Ghisellini, G., \& Tavecchio, F. 2015, MNRAS, 448, 1060

Ghisellini, G., Foschini, L., Volonteri, M., et al. 2009a, MNRAS, 399, L24

Ghisellini, G., Tavecchio, F., \& Ghirlanda, G. 2009b, MNRAS, 399, 2041

Ghisellini, G., Ceca, R. D., Volonteri, M., et al. 2010, MNRAS, 405, 387

Ghisellini, G., Tagliaferri, G., Foschini, L., et al. 2011, MNRAS, 411, 901

Ghisellini, G., Sbarrato, T., Tagliaferri, G., et al. 2014, MNRAS, 440, L111

Ghisellini, G., Haardt, F., Ciardi, B., et al. 2015, MNRAS, 452, 3457

Harrison, F. A., Craig, W. W., Christensen, F. E., et al. 2013, ApJ, 770, 103

Kalberla, P. M. W., Burton, W. B., Hartmann, D., et al. 2005, A\&A, 440, 775

Kelly, B. C., \& Bechtold, J. 2007, ApJS, 168, 1

Komissarov, S. S., Barkov, M. V., Vlahakis, N., \& Königl, A. 2007, MNRAS, 380,51

Lister, M. L., Aller, M. F., Aller, H. D., et al. 2016, AJ, 151, 133

Marcotulli, L., Paliya, V. S., Ajello, M., et al. 2017, ApJ, 839, 96

Marscher, A. P. 1980, ApJ, 235, 386

Mattox, J. R., Bertsch, D. L., Chiang, J., et al. 1996, ApJ, 461, 396

Mortlock, D. J., Warren, S. J., Venemans, B. P., et al. 2011, Nature, 474, 616

Paliya, V. S. 2015, ApJ, 804, 74

Pushkarev, A. B., Kovalev, Y. Y., Lister, M. L., \& Savolainen, T. 2017, MNRAS, 468, 4992

Sbarrato, T., Ghisellini, G., Nardini, M., et al. 2013, MNRAS, 433, 2182

Sbarrato, T., Ghisellini, G., Nardini, M., et al. 2016, MNRAS, 462, 1542

Shakura, N. I., \& Sunyaev, R. A. 1973, A\&A, 24, 337

Shaw, M. S., Romani, R. W., Cotteret, G., et al. 2012, ApJ, 748, 49

Shen, Y., Richards, G. T., Strausset, M. A., et al. 2011, ApJS, 194, 45

Tagliaferri, G., Ghisellini, G., Perri, M., et al. 2015, ApJ, 807, 167

Volonteri, M. 2010, A\&ARv, 18, 1049 